\title{
WEAKLY PRIME ONE-SIDED IDEALS
}

\author{
ANDRIES P. J. VAN DER WALT
}

(Received 25 May 1983)

Communicated by R. Lidl

\begin{abstract}
A left ideal $P$ in a ring is weakly prime if $L, K \supseteq P$ and $L K \subseteq P$ for left ideals $L$ and $K$ imply $L=P$ or $K=P$. A prime left ideal is weakly prime but the converse is false. Characterizations of weakly prime left ideals as well as a number of their properties are obtained. The intersection of all the weakly prime left ideals in a ring is a left ideal which in general is contained in (but not equal to) the prime radical.
\end{abstract}

1980 Mathematics subject classification (Amer. Math. Soc.): 16 A 66.

As in the case of a two-sided ideal, a left ideal $L$ in a ring $A$ is called prime if its complement $A \backslash L$ is an $m$-system. (See for example Dauns [1] for an extensive bibliography about prime one-sided ideals.) However, unlike in the two-sided case, if $M$ is an $m$-system in $A$ and $P$ is a left ideal which is maximal with respect to the property of not meeting $M$, then $P$ need not be prime. This is not to say that such a left ideal has no special properties; indeed, it has the very special property that if $L_{1}$ and $L_{2}$ are left ideals properly containing $P$, then $L_{1} L_{2}$ is not contained in $P$. It is the purpose of this paper to explore some properties of left ideals characterized by this condition, and which we call weakly prime.

In Section 1 we present a number of characterizations of weakly prime left ideals. Since a two-sided ideal is weakly prime if and only if it is prime, those conditions that characterize left ideals which are not two-sided are of most interest. One such is that $P$ is weakly prime if and only if it is the largest left ideal of the ring contained in its idealizer $I(P)$. Also, we introduce the concept of a weak $m$-system which plays the same part in relation to weakly prime left ideals as the concept of an $m$-system plays in relation to prime ideals.

Financial support by the Council for Scientific and Industrial Research is gratefully acknowledged. (C) 1985 Australian Mathematical Society $0263-6115 / 85 \$ A 2.00+0.00$ 
Section 2 is devoted to some miscellaneous results about weakly prime left ideals. The main result here is that a ring in which every left ideal is weakly prime is simple, which improves a result of Koh [2].

In Section 3 we define the left weak radical $w(A)$ of the ring $A$. We show that, if $A$ is left noetherian, then $w(A)$ is not only nilpotent, but that is possesses a much stronger type of nilpotence: If $K$ is any left ideal of $A$, then there is an integer $n$ such that $(w(A)+K)^{n} \subseteq K$.

\section{Definition and some characterizations}

Let $A$ be a ring with identity.

1.1 Proposition. The following are equivalent for a left ideal $P$ :

(a) $L_{1}, L_{2} \supseteq P$ and $L_{1} L_{2} \subseteq P$ imply $L_{1}=P$ or $L_{2}=P$.

(b) $\left(P+L_{1}\right)\left(P+L_{2}\right) \subseteq P$ implies $L_{1} \subseteq P$ or $L_{2} \subseteq P$.

(c) $L_{1} \supseteq P$ and $L_{1} L_{2} \subseteq P$ imply $L_{1}=P$ or $L_{2} \subseteq P$.

(d) $\left(P+L_{1}\right) L_{2} \subseteq P$ implies $L_{1} \subseteq P$ or $L_{2} \subseteq P$.

(e) $(a+P) A(b+P) \subseteq P$ implies $a \in P$ or $b \in P$.

Here $L_{1}, L_{2}$ denote left ideals and $a$, b elements of $A$.

Proof. The implications (a) $\Rightarrow$ (b) $\Rightarrow$ (c) $\Rightarrow$ (d) are immediate. So suppose (d) holds and let us prove (e). If $(a+P) A(b+P) \subseteq P$, then $a A b \subseteq P$ which implies $P A b \subseteq P$. Also, $A a A b \subseteq P$. It follows that $(A a+P)(A b) \subseteq P$, and so, by (d), $a \in P$ or $b \in P$. To conclude the proof, suppose (e) holds and let $L_{1}, L_{2} \supseteq P$ and $L_{1} L_{2} \subseteq P$. If $L_{1} \neq P$, choose $a \in L_{1} \backslash P$. Then $(A a+P)(A b+P) \subseteq P$ for every $b \in L_{2}$. This means that $(a+P) A(b+P) \subseteq P$, whence by (e), $b \in P$. So $L_{2} \subseteq P$.

We shall call a proper left ideal $P$ characterized by Proposition 1.1 weakly prime. It is easily seen that a prime left ideal is weakly prime. Moreover, a (two-sided) ideal is prime if and only if it is weakly prime, which means that in commutative rings this concept offers nothing new. On the other hand, in a noncommutative ring there are in general weakly prime left ideals which are not prime:

1.2 EXAMPLE. Let $R$ be any ring with identity which is not a prime ring, and set $A=M_{2}(R)$, the ring of all $2 \times 2$ matrices over $R$. Then the left ideal $P=R e_{11}+$ $R e_{21}$ is weakly prime. Indeed, since $P A=A$, if $L_{1}, L_{2} \supseteq P$, then $L_{1} L_{2}=L_{2}$. 
However, $P$ is not prime because, as is well known, in that case the largest two-sided ideal in $P$, namely 0 , would have to be prime, which it is not.

In view of the above remarks it is of interest to have characterizations of weakly prime left ideals which are not two-sided.

1.3 Proposition. For a left ideal $P$ which is not two-sided the following are equivalent:

(a) $P$ is weakly prime.

(b) $P L \subseteq P$ for a left ideal $L$ implies $L \subseteq P$.

(c) $P A b \subseteq P$ implies $b \in P$.

(d) $P$ is the largest left ideal of $A$ which is contained in the idealizer of $P$.

Proof. (a) $\Rightarrow$ (b): Suppose $P$ is weakly prime and let $P L \subseteq P$. then $(P A) L \subseteq P$, and, since $P$ is properly contained in $P A$ because it is not two-sided, $L \subseteq P$ follows from 1.1(c).

(b) $\Rightarrow$ (c). This is clear.

(c) $\Rightarrow$ (d): If $P \subseteq L \subseteq I(P)$, where $L$ is a left ideal of $A$ and $I(P)$ is the idealizer of $P$, then $P A b \subseteq P$ for every $b \in L$, so $L \subseteq P$. Therefore $P$ is maximal in $I(P)$. But, of course, the sum of all the left ideals of $A$ which are contained in $I(P)$ is also in $I(P)$. This establishes (d).

(d) $\Rightarrow$ (a): Assume (d) and suppose $\left(P+L_{1}\right) L_{2} \subseteq P$ for left ideals $L_{1}$ and $L_{2}$. Then $P L_{2} \subseteq P$, so $L_{2} \subseteq I(P)$, which implies $P+L_{2} \subseteq I(P)$. Therefore $L_{2} \subseteq P$ and $P$ is weakly prime by $1.1(\mathrm{~d})$.

We next turn to the relation between weakly prime ideals and $m$-systems. Recall that an $m$-system is a nonempty subset $M$ of $A$ such that for any $m_{1}, m_{2} \in M$ there is an $x \in A$ with $m_{1} x m_{2} \in M$.

1.4 Proposition. Suppose $L$ is a left ideal and $M$ is an $m$-system such that $L \cap M=\varnothing$. Then $L$ is contained in a weakly prime left ideal $P$ such that $P \cap M=\varnothing$.

Proof. In fact, if $P$ is a maximal left ideal containing $L$ and not meeting $M$, then $P$ is weakly prime: If $L_{1}, L_{2}$ properly contain $P$, then there are $m_{i} \in M \cap L_{i}$, $i=1,2$, and $x \in A$ such that $m_{1} x m_{2} \in L_{1} L_{2} \cap M$, so $L_{1} L_{2} \nsubseteq P$.

1.5 EXAMPLE. Take, in Example 1.2, $R$ to be the ring of integers modulo 9. Put $M=\left\{e_{11}+\overline{3} e_{12}\right\}$. Then $M$ is multiplicatively closed, hence an $m$-system. The 
left ideal $P$ of 1.2 is then a maximal left ideal containing $P$ and not meeting $M$, but, as was remarked, it is not prime.

Our next result gives necessary and sufficient conditions for the procedure of Proposition 1.4 to produce not only a weakly prime, but, in fact, a prime left ideal. Let us agree to denote by $\beta(L)$ the largest two-sided ideal contained in the left ideal $L$, that is, $\beta(L)=\{x \in L: x A \subseteq L\}$.

1.6 Proposition. A left ideal $P$ is prime if and only if there is an m-system $M$ such that $P$ is a maximal left ideal not meeting $M$ and $\beta(P)$ is a maximal two-sided ideal not meeting $M$.

Proof. Suppose $P$ is prime. Then $M=A \backslash L$ is an $m$-system and clearly $P$ is a maximal left ideal not meeting $M$ and $\beta(P)$ is a maximal two-sided ideal not meeting $M$.

Conversely, let $M$ be any $m$-system, let $P$ be a maximal left ideal not meeting $M$ and let $\beta(P)$ be a maximal two-sided ideal not meeting $M$. We show that $P$ is prime. Suppose $L_{1} L_{2} \subseteq P$ with $L_{1} \nsubseteq P, L_{2} \nsubseteq P, L_{1}$ and $L_{2}$ left ideals. Then we have $\left(L_{1} A+\beta(P)\right) A\left(P+L_{2}\right) \subseteq P$. Now $L_{1} A+\beta(P)$ is a two-sided ideal properly containing $\beta(P)$, so there is $m_{1} \in\left(L_{1} A+\beta(P)\right) \cap M$. Also, by the maximality of $P$, there is $m_{2} \in\left(P+L_{2}\right) \cap M$. However, this implies $\left(L_{1} A+\right.$ $\beta(P)) A\left(P+L_{2}\right) \nsubseteq P$, a contradiction; therefore $L_{1} L_{2} \nsubseteq P$ and $P$ is prime.

Results analogous to the interplay between $m$-systems and prime ideals [3] can be obtained by introducing the concept of a weak m-system: a pair $(L, M)$, where $L$ is a left ideal, $M$ is a non-empty subset of $A$ and $L \cap M=\varnothing$, is called a weak $m$-system if $[(m+L) A(n+L)] \cap M \neq \varnothing$ for all $m, n \in M$.

Observe that if $M$ is an $m$-system, then $(L, M)$ is a weak $m$-system for every left ideal $L$ such that $L \cap M=\varnothing$. Also, if $(L, M)$ is any weak $m$-system, then so is $\left(L^{\prime}, M\right)$ for any left ideal $L^{\prime}$ such that $L^{\prime} \supseteq L$ and $L^{\prime} \cap M=\varnothing$. Moreover, as a direct consequence of $1.1(\mathrm{e})$ we have the following:

1.7 Proposition. A left ideal $P$ is weakly prime if and only if $(P, A \backslash P)$ is a weak m-system.

1.8 Proposition. Let $(L, M)$ be a weak $m$-system. If $P$ is a left ideal which is maximal with respect to containing $L$ and not meeting $M$, then $P$ is weakly prime.

Proof. If $L_{1}$ and $L_{2}$ are left ideals properly containing $P$, then there are $m_{i} \in L_{i} \cap M, i=1,2$. Now $\left(L_{1} L_{2}\right) \cap M \supseteq\left[\left(m_{1}+L\right) A\left(m_{2}+L\right)\right] \cap M \neq \varnothing$ since $(L, M)$ is a weak $m$-system. Therefore $L_{1} L_{2} \nsubseteq P$, and $P$ is weakly prime. 


\section{Some results involving weak primality}

In Proposition 1.3(d) a condition for weak primality involving the idealizer is given. The next result shows another relationship between a weakly prime ideal $P$ and its idealizer, $I(P)$.

2.1 Proposition. If $P$ is a weakly prime left ideal, then $I(P)=I\left(P^{n}\right)$ for all $n \geqslant 1$.

Proof. If $P$ is two-sided there is nothing to prove, so assume $P$ is not two-sided. Suppose it is known that $I(P)=I\left(P^{k}\right)$ for some $k \geqslant 1$, and let $a \notin I(P)$. Then $P a \nsubseteq P$, and therefore $P a \nsubseteq I(P)$, by $1.3(\mathrm{~d})$. Then $P^{k+1} a=$ $P^{k}(P a) \nsubseteq P^{k}$, and since $P^{k+1} \subseteq P^{k}$, it follows that $I\left(P^{k+1}\right) \subseteq I(P)$. This concludes the proof, the other inclusion being true for any left ideal $P$.

2.2 COROLlaRY. If the weakly prime left ideal $P$ is not two-sided, then $P^{n}$ is not two-sided for any $n$.

Recall that if $P$ is a prime left ideal which is not two-sided then $\beta(P)$ is a prime ideal. Therefore $P^{n} \nsubseteq \beta(P)$ for all $n \geqslant 1$. The following proposition shows that this is actually a consequence of $P$ being weakly prime, and not so much of $\beta(P)$ being prime.

2.3 Proposition. If $P$ is a weakly prime left ideal which is not two-sided, then $P$ is not nilpotent modulo $\beta(P)$.

Proof. Let $y \notin I(P)$. An induction argument shows that $P^{n} y \nsubseteq P$ for all $n$, since if $P^{n} y \subseteq P, n \geqslant 2$, then $P\left(P^{n-1} y\right) \subseteq P$, which would mean $P^{n-1} y \subseteq P$, because $P$ is weakly prime. But then $P^{n} y \nsubseteq \beta(P)$, and hence $P^{n} \nsubseteq \beta(P)$.

2.4 COROllary. A weakly prime left ideal which is not two-sided is not nilpotent.

In [2] it was proved that a ring $A$ is simple if and only if every left ideal of $A$ is prime. Our next result strengthens this theorem in one direction.

2.5 Proposition. If every proper left ideal of $A$ is weakly prime, then $A$ is simple.

Proof. We need two preliminary observations. Firstly, if $B$ is any two-sided ideal and $L$ is any left ideal, then either $L \subseteq B$ or $B \subseteq L$, since, otherwise, 
$B L \subseteq B \cap L$ would imply that $B \cap L$ is not weakly prime. Secondly, if $B \supseteq L$, then $B L=L$, because $B L$ is weakly prime. Now suppose $B$ is any proper nonzero two-sided ideal of $A$, and let $x \in B$ be nonzero. Consider the left ideal $B x$. There are two possibilities:

(i) $x \in B x$. In this case there is $b \in B$ such that $x=b x$, that is, $(1-b) x=0$, so $l(x)$, the left annihilator of $x$, is nonzero. But $l(x) \nsubseteq B$, because $1-b \notin B$, and therefore, by the first observation above, $B \subseteq l(x)$, a contradiction.

(ii) $x \notin B x$. Put $L=A x$. Then $L \subseteq B$, and, by the second observation, $B L=L$. But this is again a contradiction, since $B L=B(A x) \subseteq B x \neq L$ because $x \in L$.

The net result of all this is that there is no proper nonzero ideal in $A$, so $A$ is simple.

\section{The left weak radical}

In this section we consider some properties of the left weak radical $w(A)$ of the ring $A$, which is defined to be the intersection of all the weakly prime left ideals in A.

3.1. Proposition. $w(A)$ is the set of all $a \in A$ such that for every weak m-system $(L, M), a \notin M$

PROOF. This follows easily from 1.7 and 1.8 .

Another characterization of $w(A)$ employs a concept which is a variant of strong nilpotence: An element $a \in A$ is called strongly nilpotent with respect to a left ideal $L$ provided every sequence $a_{0}, a_{1}, a_{2}, \ldots$ such that $a_{0}=a, a_{i+1} \in\left(a_{i}+\right.$ $L) A\left(a_{i}+L\right)$ contains an element of $L$.

3.2 Proposition. $w(A)$ is the set of all $a \in A$ such that $a$ is strongly nilpotent with respect to every left ideal in $A$.

Proof. Suppose $a \notin w(A)$. Then there is a weakly prime left ideal $P$ such that $a \notin P$. Set $a_{0}=a$; then $\left(a_{0}+P\right) A\left(a_{0}+P\right) \nsubseteq P$, and therefore there is $a_{1} \in\left(a_{0}\right.$ $+P) A\left(a_{0}+P\right)$ such that $a_{1} \notin P$. Carrying on in this way we find $a_{n+1} \in\left(a_{n}+\right.$ P) $A\left(a_{n}+P\right)$ such that $a_{n+1} \notin P$ for all $n \geqslant 0$. This means that $a$ is not strongly nilpotent with respect to $P$.

Conversely, suppose $a$ is not strongly nilpotent with respect to some left ideal $L$. Then there is a sequence $a_{0}, a_{1}, a_{2}, \ldots$ such that $a_{0}=a, a_{n+1} \in\left(a_{n}+\right.$ $L) A\left(a_{n}+L\right)$ with $a_{n} \notin L$ for all $n \geqslant 0$. Let $P$ be a maximal left ideal containing 
$L$ and not meeting $M=\left\{a_{n}\right\}$; we shall prove that $P$ is weakly prime. Indeed, suppose $L_{1}$ and $L_{2}$ are left ideals which properly contain $P$. Then there are $a_{i_{j}} \in L_{j} \cap M, j=1,2$. Let $m=\max \left(i_{1}, i_{2}\right)$. Then $a_{m} \in L_{1}$ and $a_{m} \in L_{2}$, because if $a_{k} \in K$ for some left ideal $K \nsupseteq L$, then also $a_{k+1} \in K$. Therefore $a_{m+1} \in L_{1} L_{2}$ and so $L_{1} L_{2} \cap M \neq \varnothing$. This means that $L_{1} L_{2} \nsubseteq P$, and $P$ is weakly prime, so that $a \notin w(A)$.

The left weak radical is certainly not a radical in the usual sense of the word. For one thing, it need not be a two-sided ideal, as the following example due to Schultz [4] shows.

3.3 ExAmple. Let $F$ be a field and consider the ring $A$ of all matrices of the form

$$
\left[\begin{array}{ccc}
f_{1} & 0 & 0 \\
f_{2} & a & f_{4} \\
f_{3} & 0 & c
\end{array}\right]
$$

where $f_{1}, f_{2}, f_{3}, f_{4}$ and $a$ are arbitrary elements of $F$. It is not too difficult to show that $w(A)=F e_{23}$, which is not two-sided.

This example also shows that $w(A)$ may be properly contained in the prime radical $\operatorname{rad}(A)$ of $A$. Of course, every prime ideal being weakly prime, $w(A) \subseteq$ $\operatorname{rad}(A)$ is always true.

3.4 Proposition. Suppose $A$ is left noetherian. If $K$ is any left ideal of $A$, then there exists $n>0$ such that $(w(A)+K)^{n} \subseteq K$. Moreover, $w(A)$ contains every left ideal with this property.

Proof. We prove the last assertion first. Suppose $L$ is a left ideal with the property that, given a left ideal $K$, there exists an integer $n$ such that $(L+K)^{n} \subseteq$ $K$. Let $P$ be any weakly prime left ideal. If $L \nsubseteq P$, then $(L+P)$ properly contains $P$, and therefore $(L+P)^{n} \nsubseteq P$. So $L \subseteq P$ and hence $L \subseteq w(A)$. To prove the first assertion, suppose there is a left ideal $K$ such that $\left(w^{\prime}(A)+K\right)^{n} \nsubseteq K$ for all $n>0$. Let $K_{0}$ be a maximal such left ideal. Then certainly $w(A) \nsubseteq K_{0}$, and we shall show that this is a contradiction by proving that $K_{0}$ is weakly prime. Indeed, if $L_{1}, L_{2}$ are left ideals properly containing $K_{0}$, then, by the maximality of $K_{0}$, there are $m_{i}$ such that $\left(w(A)+L_{i}\right)^{m_{i}} \subseteq L_{i}, i=1,2$. But then $(w(A)+$ $\left.K_{0}\right)^{m_{1}+m_{2}} \subseteq\left(w(A)+L_{1}\right)^{m_{1}}\left(w(A)+L_{2}\right)^{m_{2}} \subseteq L_{1} L_{2}$, so $L_{1} L_{2} \nsubseteq K_{0}$, and $K_{0}$ is weakly prime. 
Exactly what the structural significance of $w(A)$ is, remains to be seen. A first result in this connection is the following.

3.5 Proposition. Suppose $A=L_{1} \oplus L_{2} \oplus \cdots \oplus L_{n}$ where the $L_{i}$ are left ideals such that $L_{i} A=A, i=1, \ldots, n$. Then $w(A)=0$.

Proof. If $L A=A$ for a left ideal $L$, then $L$ is weakly prime.

3.6 Corollary. Let $R$ be any ring with identity, and let $A=M_{n}(R)$ be the ring of $n \times n$ matrices over $R$. Then $w(A)=0$.

\section{Acknowledgement}

Helpful discussions with Talvin Schultz and Hennie Schutte are gratefully acknowledged.

\section{References}

[1] J. Dauns, 'Prime modules and one-sided ideals', Ring theory and algebra III, pp. 301-344 (Lecture Notes in Pure and Applied Mathematics 55, Marcel Dekker, 1980).

[2] K. Koh, 'On prime one-sided ideals', Canad. Math. Bull. 14(2) (1971), 259-260.

[3] N. H. McCoy, 'Prime ideals in general rings', Amer. J. Math. 71 (1949), 823-833.

[4] T. G. Schultz, Private communication.

Department of Mathematics

University of Stellenbosch

Stellenbosch

Republic of South Africa 